\title{
Clinical Performance of ADNEX (The Assessment of Different NEoplasias in the adneXa) Model in Early Diagnosis and Staging of Benign and Malignant Ovarian Tumors
}

\author{
Jumei Hu, Yushuang Shi, Mengxiong Li, Cunjian Yi* \\ Department of Obstetrics and Gynecology, The First Affiliated Hospital of Yangtze University, Jingzhou, China \\ Email: *Cunjiany@163.com
}

How to cite this paper: Hu, J.M., Shi, Y.S., Li, M.X. and Yi, C.J. (2017) Clinical Performance of ADNEX (the Assessment of Different NEoplasias in the adneXa) Model in Early Diagnosis and Staging of Benign and Malignant Ovarian Tumors. Yangtze Medicine, 1, 148-156.

https://doi.org/10.4236/ym.2017.13015

Received: May 19, 2017

Accepted: September 18, 2017

Published: September 21, 2017

Copyright $\odot 2017$ by authors and Scientific Research Publishing Inc. This work is licensed under the Creative Commons Attribution International License (CC BY 4.0).

http://creativecommons.org/licenses/by/4.0/

\begin{abstract}
Objective: To investigate the clinical value of ADNEX model in early diagnosis and staging of benign and malignant ovarian tumors. Method: 136 cases of ovarian cancer patients treated in our hospital were retrospectively analyzed using the ADNEX risk model and MRI data. The accuracy of the two diagnostic methods was compared with the results of pathological examination as gold standard. Results: For qualitative assessment, the accuracy and sensitivity of the ADNEX model were $78.70 \%$ and $93 \%$, while the accuracy and sensitivity of MRI examination were $80.1 \%$, and $90.7 \%$, respectively. The diagnostic values of the two methods were not statistically different $(P>0.05)$. For ovarian tumor staging, the ADNEX model was significantly less accurate and specific for staging borderline tumor than MRI examination, although it had significantly higher sensitivity $(P<0.05)$. For tumors at other stages, there were no diagnostic differences between the methods $(P>0.05)$. Conclusion: ADNEX risk model has certain diagnostic and predictive value to distinguish benign from malignant ovarian tumors. It is useful to detect and exclude ovarian tumor. However, for early diagnosis, it is not accurate enough and further study is needed to validate this usefulness.
\end{abstract}

\section{Keywords}

Ovarian Cancer, ADNEX Risk Model, MRI Examination

\section{Introduction}

The incidence of ovarian cancer ranks the third in gynecological malignancies with the highest mortality [1]. Early diagnosis and cytoreductive surgery im- 
prove 5-year survival rate [2] [3]. However, early screening and diagnosis of ovarian cancer is a hot but difficult spot in ovarian cancer research. The auxiliary diagnosis of ovarian cancer mainly includes the use of imaging examination and serum markers. Since ultrasound examination is simple and cost effective, it is most widely used in gynecological examination. To maximize the efficiency of early diagnosis of ovarian cancer, a number of ultrasound models have been proposed [4] [5] [6]. In 2014, the Assessment of Different NEoplasias in the adneXa (ADNEX) model was proposed to differentiate between benign, borderline, early and advanced stage invasive, and secondary metastatic tumors [7]. It can automatically provide differentiation between benign and malignant and tumor staging information on mobile devices or websites using clinical information and ultrasound data. At present, the clinical performance of the model has not been reported in China. The aim of this study is to investigate the clinical value of the model in the early diagnosis and staging of benign and malignant ovarian tumors.

\section{Subjects and Methods}

\subsection{Subjects}

223 cases of patients enrolled at the First Affiliated Hospital of Yangtze University from January 2011 to October 2015 with ovarian cancer were retrospectively analyzed. The patients were preoperatively diagnosed using color Doppler ultrasound and postoperatively confirmed pathologically to have epithelial tumors. Of them, 136 patients had ultrasound data for the ADNEX modeling and were examined using pelvic MRI examination. Among them, there were benign in 93 cases and malignant in 43 cases. The age ranged from 19 to 74 years with an average age of 45.6 years. MRI and pathological staging were preformed based on 2013 FIGO [8]. Inclusive criteria: 1) From January 2011 to October 2015, we have admitted ovarian cancer to the Department of Obstetrics and gynecology in our hospital; 2) Histopathological diagnosis of ovarian tumors is clear, and the nature and pathological staging of ovarian tumors are determined; 3) Ovarian tumors are epithelial; 4) All the patients were examined by transvaginal ultrasound before operation. The ultrasonic image data can be read out or recorded, and all the index data needed for the ADNEX model can be read out; 5) MRI examination was performed before or after operation, with or without abdominal distension, abdominal pain and other clinical symptoms; 6) Preoperative serum CA125 examination is available or not. Exclusion criteria: 1) No MRI examination was performed before the operation; 2) Non epithelial ovarian tumor; 3) The ultrasonic inspection record is incomplete or missing image; 4) Exclusion of endometriosis, tuberculous peritonitis, tumors outside the reproductive tract (retroperitoneal neoplasms, rectal cancer, sigmoid colon cancer, etc.).

\subsection{Methods}

Ultrasound examinations were conducted using Philips ClearVue 580 system 
and reported by the same physician. The married patients were examined by transvaginal examination, while the unmarried patients were examined by rectal examination. If the tumors were large, transabdominal examinations were preformed.

MRI axial, sagittal and coronal scans were preformed using GE Signal $1.5 \mathrm{~T}$ magnetic resonance imaging system. The field of view (FOV) was $28-36 \mathrm{~cm}$ and the layer thickness/spacing were $5 \mathrm{~mm} / 1 \mathrm{~mm}$. T1WI was obtained using SE sequence at TR/TE: $350-550 / 10 \mathrm{~ms}$, with a matrix of $256 \times(192-128)$. The number of acquisition was 2. T2WI was generated using FSE sequence at TR/TE: $3000 / 108 \mathrm{~ms}$ with a matrix of $320 \times 224$. The number of scans was 4 . The enhanced scanning was preformed once at TR/TE: 80 to $150 \mathrm{~ms} /$ minimum with a matrix of $256 \times(192-224)$. The number of scans was 1 . The contrast agent was acyclic, ionic gadolinium (GD-DTPA), used at a dose of 0.1 to $0.2 \mathrm{mmol} / \mathrm{kg}$, injected at a rate of $2.5 \mathrm{ml} / \mathrm{s}$ rate through elbow vein.

Serum CA125 was detected using ADVIA Centaur XP automated chemiluminescence analysis system and associated kit (Siemens, Germany).

ADNEX modeling used 3 clinical indexes such as age, serum CA125 level and category of diagnosis and treatment center (whether the medical institutions had tumor diagnosis center) and 6 ultrasound parameters such as the maximum diameter of lesion, ratio of solid tissue, whether there were more than 10 cysts, the number of papillae, whether there were echoes or not and whether there were ascites or not. The sampling of images acquired with ultrasound is shown in Figures 1(a)-(f). ADNEX model was accessed at http://www.iotagroup.org/adnexmodel/. Once relevant data were input, the property and staging of the tumor were automatically generated by the on-line ADNEX model software.
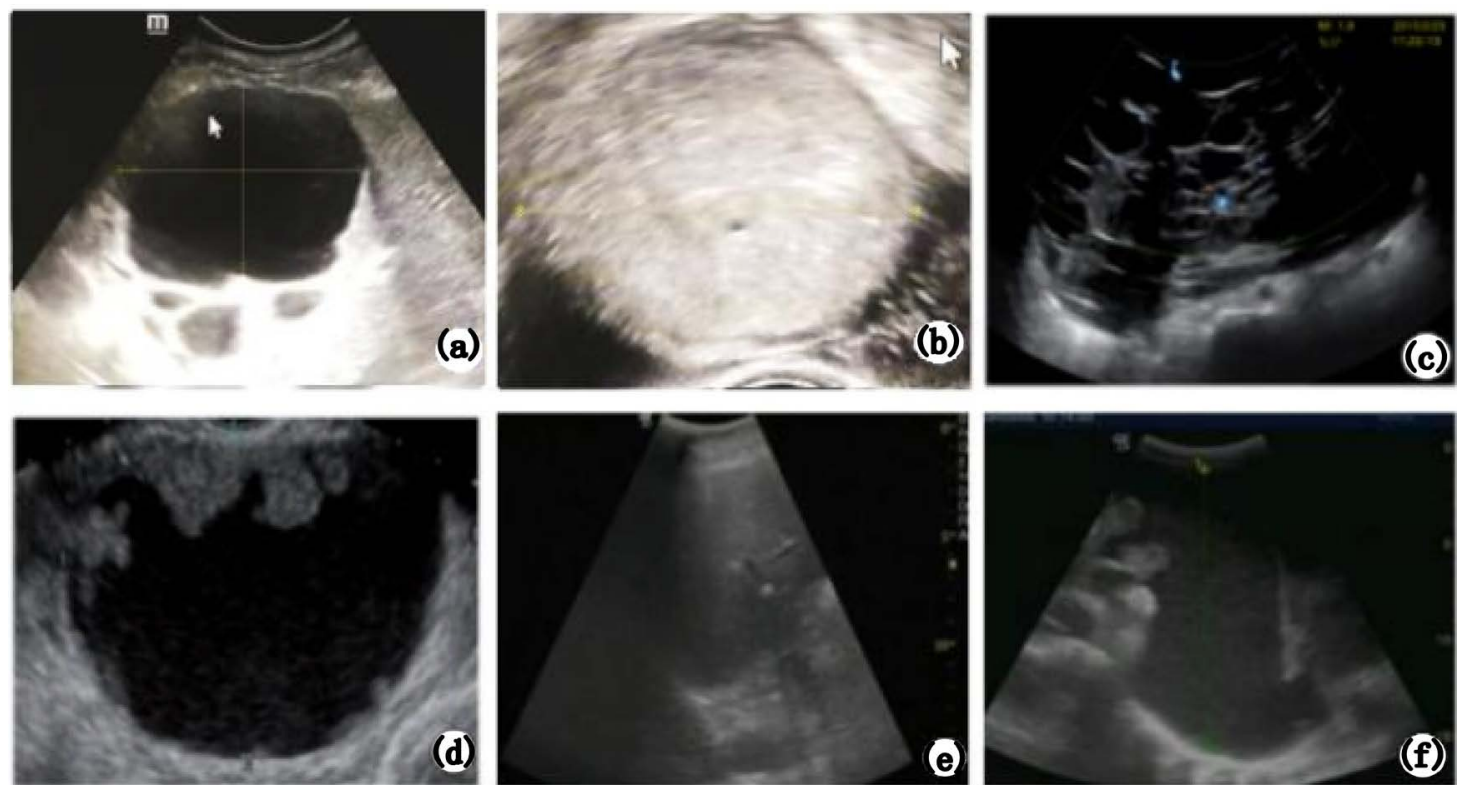

Figure 1. Collecting indicator for ADNEX model. (a) The maximum diameter of tumor (mm); (b) Measurement of solid tissue; (c) Count of cysts to see if they are more than 10; (d) The number of papillae $(0,1,2,3,>3)$; (e) Echo or not; (f) Ascites or not. 


\subsection{Statistical Analysis}

Data were processed using SPSS 17.0 statistical software. Enumeration data were tested using $X^{2}$ test. The data were considered statistically different when $P$ is $<0.05$ and were tested using a receiver operating characteristic curve (ROC).

\section{Results}

\subsection{Distinguishment of Benign and Malignant Ovarian Tumors}

Among the 136 cases, 93 and 43 (including were classified as benign and malignant), (including borderline malignant) based on FIGO (2013), respectively. Based on the ADNEX model software, 70 cases were benign, and 66 cases were malignant. The accuracy, sensitivity and specificity of the ADNEX model were $78.7 \%$, $93 \%$, and $72 \%$, respectively, as compared to the FIGO system. The positive and negative predictive values were $60.6 \%$ and $95.7 \%$, respectively (Table 1).

\subsection{Staging of Ovarian Cancer by the ADNEX Model}

Compared to the pathological results, the ADNEX model classified the tumors into five stages benign, borderline, I stage, II to IV stage and metastatic tumor (Table 2).

Table 1. The outcome of the ADNEX modeling on benign and malignant ovarian tumors.

\begin{tabular}{cccc}
\hline & \multicolumn{2}{c}{ ADNEX modeling } & \multirow{2}{*}{ Total } \\
\cline { 2 - 3 } Pathological examination & Malignant & Benign & \\
\cline { 2 - 3 } Malignant & 40 & 3 & 43 \\
Benign & 26 & 67 & 93 \\
Total & 66 & 70 & 136 \\
\hline
\end{tabular}

Table 2. Prediction of tumor stage using the ADNEX model.

\begin{tabular}{cccccc}
\hline & Benign & Borderline & I stage & $\begin{array}{c}\text { II to IV } \\
\text { stage }\end{array}$ & $\begin{array}{c}\text { Metastatic } \\
\text { tumor }\end{array}$ \\
\hline Accuracy (\%) & 78.6 & 86.0 & 91.9 & 86.8 & 93.4 \\
Sensitivity (\%) & 72.0 & 50.0 & 25.0 & 90.9 & 14.3 \\
Specificity (\%) & 93.0 & 87.8 & 96.1 & 86.0 & 97.7 \\
Positive predictive value (\%) & 95.7 & 15.8 & 28.6 & 55.6 & 25.0 \\
Negative predictive value (\%) & 60.6 & 97.4 & 95.3 & 98.0 & 95.5 \\
\hline
\end{tabular}

Remark: ACC: Accuracy; SENS: Sensitivity, SPEC: Specificity; PPV: Positive predictive value; NPV: Negative predictive value. 


\subsection{Comparison of the ADNEX Model and MRI in the Diagnosis of Benign and Malignant Ovarian Tumors}

Use the pathological results as gold standard, MRI detected 74 cases of benign and 62 cases of malignant tumors. The ROC analysis showed that the areas under the curve (AUC) in the ADNEX model and MRI data were 0.825 and 0.830, respectively (Figure 2). Statistical analysis showed that there were no significant difference between the two methods in the accuracy, sensitivity, specificity, positive predictive value and negative predictive value $(P>0.05$, Table 3$)$.

The AUC is 0.825 and 0.830 for the ADNEX model and MRI, respectively.

\subsection{Comparison of the ADNEX Model and MRI in Tumor Staging}

Using the pathological results as gold standard, the ADNEX model detected 70

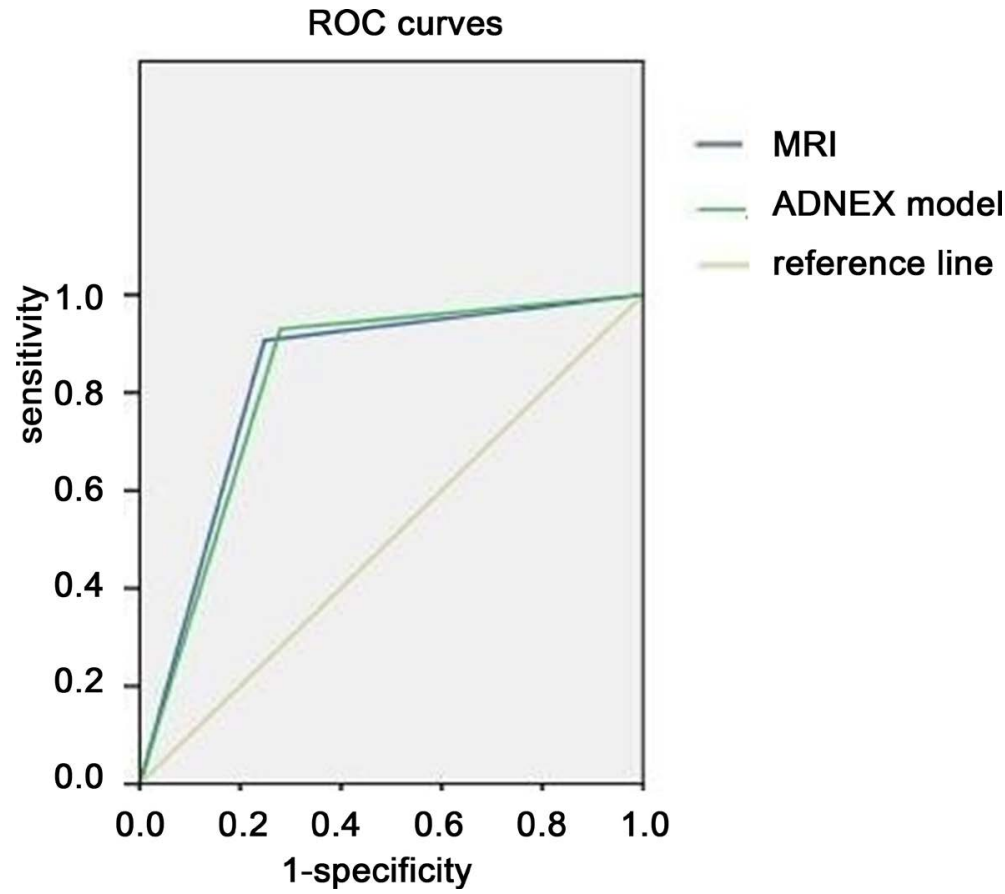

Figure 2. ROC showing the comparison of the ADNEX model and MRI in the detection of benign and malignant ovarian tumors.

Table 3. Comparison of the ADNEX model and MRI in differentiating benign and malignant ovarian tumors.

\begin{tabular}{ccccc}
\hline & ADNEX model & MRI & $\mathrm{X}^{2}$ & $\mathrm{P}$ \\
\hline Accuracy (\%) & 78.7 & 80.1 & 0.09 & 0.764 \\
Sensitivity (\%) & 93.0 & 90.7 & 0.156 & 0.693 \\
Specificity (\%) & 72.0 & 75.3 & 0.249 & 0.618 \\
Positive predictive value (\%) & 60.6 & 62.9 & 0.071 & 0.789 \\
Negative predictive value (\%) & 95.7 & 94.6 & 0.098 & 0.755 \\
\hline
\end{tabular}


benign, 19 borderline, 7 stage I, 36 stage II - IV and 4 metastatic tumors. MRI detected 74, 0 borderline, 18 stage I, 43 stage II- IV and 1 metastatic tumors. Compared with MRI, the ADNEX model has significantly lower accuracy and specificity, but significantly higher sensitivity for borderline tumor $(P<0.05)$. For other diagnostic outcomes, the results were similar between the two methods $(P>0.05$, Table 4$)$.

Table 4. Comparison of the ADNEX model and MRI in staging ovarian tumors.

\begin{tabular}{|c|c|c|c|c|c|}
\hline Tumor stage & Diagnostic value & ADNEX model & MRI & $\mathrm{X}^{2}$ & $P$ \\
\hline \multirow{5}{*}{ Benign } & Accuracy (\%) & 78.6 & 80.1 & 0.09 & 0.764 \\
\hline & Sensitivity (\%) & 72.0 & 75.3 & 0.249 & 0.618 \\
\hline & Specificity (\%) & 93.0 & 90.7 & 0.156 & 0.693 \\
\hline & Positive predictive value (\%) & 95.7 & 94.6 & 0.098 & 0.755 \\
\hline & Negative predictive value (\%) & 60.6 & 62.9 & 0.071 & 0.789 \\
\hline \multirow{5}{*}{ Borderline } & Accuracy (\%) & 86.0 & 95.6 & 7.44 & 0.006 \\
\hline & Sensitivity (\%) & 50.0 & - & 4.000 & 0.046 \\
\hline & Specificity (\%) & 87.8 & 100 & 17.049 & - \\
\hline & Positive predictive value (\%) & 15.8 & - & - & - \\
\hline & Negative predictive value (\%) & 97.4 & 95.6 & 6.26 & 0.429 \\
\hline \multirow{5}{*}{ I stage } & Accuracy (\%) & 91.9 & 88.2 & 1.028 & 0.311 \\
\hline & Sensitivity (\%) & 25.0 & 62.5 & 2.286 & 0.131 \\
\hline & Specificity (\%) & 96.1 & 89.8 & 3.824 & 0.051 \\
\hline & Positive predictive value (\%) & 28.6 & 27.8 & 0.002 & 0.968 \\
\hline & Negative predictive value (\%) & 95.3 & 97.5 & 0.781 & 0.377 \\
\hline \multirow{5}{*}{ II to IV stage } & Accuracy (\%) & 86.8 & 78.7 & 3.112 & 0.078 \\
\hline & Sensitivity (\%) & 90.9 & 81.8 & 0.772 & 0.380 \\
\hline & Specificity (\%) & 86.0 & 78.1 & 2.409 & 0.121 \\
\hline & Positive predictive value (\%) & 55.6 & 41.9 & 1.472 & 0.225 \\
\hline & Negative predictive value (\%) & 98.0 & 95.7 & 0.847 & 0.357 \\
\hline \multirow{5}{*}{ Metastatic } & Accuracy (\%) & 93.4 & 94.1 & 0.063 & 0.802 \\
\hline & Accuracy (\%) & 14.3 & - & 1.077 & 0.299 \\
\hline & Sensitivity (\%) & 97.7 & 99.2 & 1.016 & 0.314 \\
\hline & Specificity (\%) & 25.0 & - & 0.313 & 0.576 \\
\hline & Positive predictive value (\%) & 95.5 & 94.8 & 0.059 & 0.808 \\
\hline
\end{tabular}




\section{Discussion}

Ovarian cancer is a common malignant tumor in female reproductive systems, the incidence rate ranks the third and only seconds to cervical cancer and uterine cancer. Furthermore, the incidence has been increasing recently. It has been a hot but challenging spot to find effective early diagnosis method. The advantage of the ADNEX model is that it is designed specifically for predicting and staging benign and malignant ovarian tumors in a cost effective way. It uses conventional clinical information and ultrasound data for on-line prediction, irrespective of the availability of CA125 data. For ultrasound examination, data corrected by inexperienced physician are sufficient for modeling. MRI provides images at various directions and layers, and is especially suitable for soft tissue. It can display the relationship between the various organs in the pelvic cavity and guide surgical operation although the reports may be somewhat subjective.

\subsection{The Clinical Significance of the ADNEX Model in the Diagnosis of Benign and Malignant Ovarian Tumors}

It was reported that when the two sets of data were used for the diagnosis of benign and malignant ovarian tumors, the accuracy of the ADNEX model was $79.9 \%$, and $81.3 \%$, respectively [9]. We found that the accuracy, sensitivity and specialty of the ADNEX model were $78.70 \%, 93 \%$, and $72 \%$, respectively with the positive predictive value of $60.6 \%$ and negative predictive value of $95.7 \%$. The accuracy is similar to the previous report. The accuracy, sensitivity, specialty, positive predictive value and negative predictive value of MRI were $80.1 \%$, $90.7 \%, 75.3 \%, 62.9 \%$ and $94.6 \%$, respectively. AUC was 0.825 and 0.830 for the ADNEX model and MRI, suggesting that both methods have excellent diagnosis value, although MRI is slightly better than the ADNEX model. Statistically, the two methods are the same in the tumor diagnosis. The outcomes of the ADNEX model showed that it is better for the detection and exclusion of ovarian tumors. It is clear that the ADNEX model is clinically valuable for the diagnosis of benign and malignant ovarian tumors as MRI technology.

\subsection{The Clinical Significance of the ADNEX Model in Staging Ovarian Tumors}

Traditionally, ovarian tumor staging is mainly depended on pathological examination, not on ultrasound data. The staging results based on the ADNEX model are similar to those reported previously [9]. The accuracy and sensitivity of the ADNEX model on early stage tumors were less than on late stage tumors. For early stage tumors, the ADNEX model and MRI are similar. For borderline tumor staging, the ADNEX model is less accurate and specific but more sensitive as compared with MRI $(P<0.05)$. For staging tumors at other stages, the outcomes from the two methods are slightly, and statistically insignificantly different $(P>0.05)$. Therefore, the ADNEX model is better at ovarian tumor staging, while MRI cannot directly stage the tumors, particularly for borderline tumor. 
Although the ADNEX model is not perfect but it is a big step forward in tumor staging, despite its low sensitivity to early stage tumor. For better clinical use of the ADNEX model and higher qualitative assessment and staging of benign and malignant ovarian tumors, we have identified a number of shortcomings in the ADNEX model. For example, the age input has to be $\geq 14$; the maximum diameter of tumor must be $\geq 8 \mathrm{~mm}$. It is desirable to improve the model making it possible to accommodate the data outside the current range for better applicability. In addition, parameters used in the model may be expended to include indexes describing lymph node enlargement, nodes in pelvic cavity and posterior fornix, blood flow signal and resistance if any. Finally, due to the retrospective nature of the study, the ultrasound data parameters collected did not strictly follow what are required in the model, and some of the data were estimated. The limited sample size may also affect the diagnostic efficacy of the ADNEX model and MRI examination. It is likely that the model would have better diagnosis performance for differentiating benign and malignant ovarian tumors and their staging if the model is modified, clinical data are collected according to the model requirement, and further prospective study is conducted.

In conclusion, our study shows the ADNEX model is clinically value for differentiating benign and malignant ovarian tumors and their staging. It is useful for detection and exclusion of ovarian tumors, although its staging ability for early stage tumor needs further improvement and validation.

\section{References}

[1] Jemal, A., Siegel, R., Xu, J., et al. (2010) Cancer Statistics. CA: A Cancer Journal for Clinicians, 60, 277-300. https://doi.org/10.3322/caac.20073

[2] Badgwell, D. and Bast, Jr. R.C. (2007) Early Detection of Ovarian Cancer. Disease Markers, 23, 397-410. https://doi.org/10.1155/2007/309382

[3] Liang, M.L. and Wang, Z.H. (2012) The Screening and Early Diagnosis of Ovarian Cancer. Chinese Journal of Practical Gynecology and Obstetrics, 28, 166-169.

[4] Alcazar, J.L., Guerriero, S., Laparte, C., et al. (2011) Contribution of Power Doppler Blood Flow Mapping to Grayscale Ultrasound for Predicting Malignancy of Adnexal Masses in Symptomatic and Asymptomatic Women. European Journal of Obstetrics, Gynecology, and Reproductive Biology, 155, 99-105. https://doi.org/10.1016/j.ejogrb.2010.11.010

[5] Meng, L., Shi, T.M. (2015) IOTA Simple Rules in Differentiating between Benign and Malignant Ovarian Tumors. Journal of Chinese Clinical Medical Imaging, 26, 502-504.

[6] Liu, F. (2015) Evaluation of Ultrasonic Exam in Differentiation Diagnosis of Ovarian Tumors Using Logistic Regression. Journal of Modern Oncology, 23, 264-266.

[7] Van Calster, B., Van Hoorde, K., Valentin, L., Testa, A.C., Fischerova, D., Van Holsbeke, C., et al. (2014) Evaluating the Risk of Ovarian Cancer before Surgery Using the ADNEX Model to Differentiate between Benign, Borderline, Early and Advanced Stage Invasive, and Secondary Metastatic Tumours: Prospective Multicentre Diagnostic Study. BMJ, 349, Article No: g5920. https://doi.org/10.1136/bmj.g5920

[8] Lin, Z.Q., (2013) FIGO 2013 New Stage of Ovarian Cancer, Fallopian Tube Cancer, 
Peritoneal Cancer. Chinese Journal of Practical Gynecology and Obstetrics, 29, 921-923.

[9] Szubert, S. (2016) External Validation of the IOTA ADNEX Model Performed by Two Independent Gynecologic Centers. Gynecologic Oncology, 142, 490-495.

https://doi.org/10.1016/j.ygyno.2016.06.020

\section{Scientific Research Publishing}

Submit or recommend next manuscript to SCIRP and we will provide best service for you:

Accepting pre-submission inquiries through Email, Facebook, LinkedIn, Twitter, etc.

A wide selection of journals (inclusive of 9 subjects, more than 200 journals)

Providing 24-hour high-quality service

User-friendly online submission system

Fair and swift peer-review system

Efficient typesetting and proofreading procedure

Display of the result of downloads and visits, as well as the number of cited articles

Maximum dissemination of your research work

Submit your manuscript at: http://papersubmission.scirp.org/

Or contact ym@scirp.org 